\title{
Abundância e sazonalidade de cigarrinhas (Hemiptera, Cicadellidae, Cicadellinae) em vegetação herbácea de pomar de laranja doce, no município de Montenegro, Estado do Rio Grande do Sul, Brasil
}

\author{
Ana P. Ott ${ }^{1}$, Wilson S. Azevedo-Filho ${ }^{1}$, Augusto Ferrari ${ }^{2} \&$ Gervásio S. Carvalho $^{2}$ \\ 1. Departamento de Fitossanidade, Faculdade de Agronomia, Universidade Federal do Rio Grande do Sul (UFRGS), Av. Bento Gonçalves, \\ 7712, 90540-000 Porto Alegre, RS, Brasil. (ana.ott@ufrgs.br) \\ 2. Departamento de Biologia, Faculdade de Biociências, Pontifícia Universidade Católica do Rio Grande do Sul (PUCRS), Av. Ipiranga, \\ 6681, 90619-900 Porto Alegre, RS, Brasil. (gervasio@ pucrs.br)
}

\begin{abstract}
Abundance and seasonality of leafhoppers (Hemiptera, Cicadellidae, Cicadellinae) in herbaceous vegetation of sweet orange orchard at Montenegro County, State of Rio Grande do Sul, Brazil. This paper aimed to study leafhopper's populational dynamics and constancy on herbaceous vegetation of a sweet orange orchard (Citrus sinensis (L.) Osbeck, Valencia variety) in southern Brazil. The survey was performed with sweeping net method, from February/2001 to January/2002. Monthly, 10 samples of 50 sweeps were taken in the herbaceous vegetation between orchard's lines $(n=500$ sweeps/sampling). A total of 928 individuals were collected, belonging to nine species: Bucephalogonia xanthophis (Berg, 1879), Diedrocephala variegata (Fabricius, 1775), Hortensia similis (Walker, 1851), Macugonalia leucomelas (Walker, 1851), Parathona gratiosa (Blanchard, 1840), Plesiommata corniculata Young, 1977, Sibovia sagata (Signoret, 1854), Sonesimia grossa (Signoret, 1854), and Tapajosa rubromarginata (Signoret, 1855). The highest abundance was registered in summer and spring. Hortensia similis and M. leucomelas were the only species considered constant during the survey. Leafhopper abundance was lower in the April and December, probably due to abiotic factors (rainfall) and vegetation structure (plants height) effects.
\end{abstract}

KEYWORDS. Agroecology, constancy, Citrus sinensis, populational fluctuation, sweep net.

RESUMO. O presente estudo teve por objetivo conhecer a flutuação populacional e a constância de cicadelíneos presentes na vegetação herbácea de um pomar de laranja doce (Citrus sinensis (L.) Osbeck), variedade Valência, em Montenegro, RS. O levantamento foi realizado com a metodologia da rede-de-varredura, tendo início em fevereiro de 2001 e finalizando-se em janeiro de 2002. Mensalmente foram coletadas dez amostras, sendo cada uma destas composta por 50 golpes de rede em movimento de avanço sobre a vegetação herbácea e cultura intercalar nas entrelinhas do pomar ( $\mathrm{n}=500$ golpes/ocasião de coleta). No total foram coletados 928 cicadelíneos, pertencentes a nove espécies: Bucephalogonia xanthophis (Berg, 1879), Diedrocephala variegata (Fabricius, 1775), Hortensia similis (Walker, 1851), Macugonalia leucomelas (Walker, 1851), Parathona gratiosa (Blanchard, 1840), Plesiommata corniculata Young, 1977, Sibovia sagata (Signoret, 1854), Sonesimia grossa (Signoret, 1854) e Tapajosa rubromarginata (Signoret, 1855). O período de maior abundância da comunidade de cicadelíneos foi durante a primavera e o verão. Hortensia similis e M. leucomelas foram as únicas espécies consideradas constantes durante o levantamento. A menor abundância dos cicadelíneos em abril e dezembro provavelmente devese a efeitos causados por fatores abióticos (pluviosidade) e aqueles relativos à estrutura da vegetação (altura das plantas) sobre a comunidade.

PALAVRAS-CHAVE. Agroecologia, constância, Citrus sinensis, flutuação populacional, rede-de-varredura.

A bactéria Xylella fastidiosa Wells et al., 1987, agente causal da Clorose Variegada dos Citros (CVC), é restrita ao xilema das plantas, sendo transmitida por cigarrinhas da subfamília Cicadellinae, tribos Cicadellini e Proconiini (Hemiptera, Cicadellidae) (RedAK et al., 2004). $\mathrm{Na}$ última década, a CVC tem sido apontada como responsável por perdas consideráveis na produção de laranja doce no país. Esta doença foi detectada pela primeira vez no Brasil em 1987, em pomares do noroeste paulista e, atualmente, está presente na maioria dos estados brasileiros produtores de citros (Rossetr et al., 1997), inclusive no Rio Grande do Sul.

Estudos sobre a flutuação populacional de cigarrinhas vetoras de $X$. fastidiosa em pomares cítricos têm enfocado as espécies Acrogonia citrina Marucci \& Cavichioli, 2002, Dilobopterus costalimai Young, 1977 e Oncometopia facialis (Signoret, 1854), as quais tem tido sua ocorrência e distribuição associadas a fatores abióticos como pluviosidade e temperatura (ROBERTo \& Ү АмАмото, 1998; ҮАмамото et al., 2002).
Yamamoto \& Gravena (2000), em estudo sobre a constância de 29 espécies de cicadelíneos na região Norte do Estado de São Paulo, registraram as espécies consideradas vetoras de $X$. fastidiosa como de ocorrência acidental. Nesse mesmo estado, constatou-se um padrão de ocorrência para espécies de cicadelíneos vetores de $X$. fastidiosa, que sugere a presença de um grupo com espécies exclusivas dos citros (Acrogonia sp., D. costalimai e $O$. facialis e outro com espécies que ocorrem mais freqüentemente na vegetação herbácea, tais como: Bucephalogonia xanthophis (Berg, 1879) e Macugonalia leucomelas (Walker, 1851) (PAIVA et al., 1996).

A abundância e a constância das espécies de cicadelíneos que ocorrem na vegetação herbácea nas entrelinhas e/ou áreas adjacentes aos pomares é de grande relevância, uma vez que esta vegetação pode servir como hospedeiro alternativo, provendo alimento e refúgio para a reprodução das cigarrinhas de xilema, sendo imprescindível para o seu completo desenvolvimento (HuAng \& Chiaradia, 1998; Paiva et al., 1996). Estudos 
comprovam a ocorrência de cicadelíneos vetores de $X$. fastidiosa em proporções semelhantes tanto nas plantas cítricas como na vegetação nativa no Estado de São Paulo. Sua presença é constante na vegetação herbácea, enquanto nas plantas cítricas a maioria das espécies apresentaram ocorrência acidental (YАмамото, 1996; YAMAMOTO \& GRAVENA, 2000).

Este trabalho teve como objetivo estudar a abundância e a sazonalidade de espécies de cicadelíneos presentes na vegetação herbácea e cultura intercalar de um pomar de laranja doce com manejo orgânico, reunindo dados que possam contribuir para o conhecimento da dinâmica destas populações e assim, possibilitar maior eficiência das medidas de controle e manejo destas cigarrinhas, que têm a capacidade de transmitir a bactéria $X$. fastidiosa.

\section{MATERIAL E MÉTODOS}

O estudo foi realizado em um pomar de Citrus sinensis (L.) Osbeck, variedade Valencia, com idade de dez anos, no município de Montenegro (513' $36^{\prime \prime} \mathrm{W}$ $29^{\circ} 31^{\prime} 27^{\prime \prime}$ 'S), Rio Grande do Sul. Este pomar é integrante da Cooperativa dos Citricultores Ecológicos do Vale do Caí (Ecocitrus), que usa tecnologias orgânicas de manejo (aplicação de biofertilizante e calda bordalesa). O pomar apresenta cerca de 200 árvores e caracteriza-se pela vegetação herbácea composta por gramíneas (Andropogon lateralis Nees, Paspalum notatum Fluegge, Brachiaria sp., Digitaria sp.); compostas [Baccharis coridifolia DC, B. trimera (Less.) DC, Bidens pilosa L., Vernonia sp.]; leguminosas [Desmodium tortuosum $(\mathrm{Sw})$ DC, Trifolium sp., Vicea sp.]; solanáceas (Solanum ciliatum Lam., S. auriculatum Aiton) e ciperáceas (Cyperus rotundus L.). Como cultura intercalar foi plantada batata-doce (Ipomoea batatas L.).

Realizaram-se coletas junto à vegetação herbácea e na cultura intercalar do pomar com uma rede-devarredura de seção circular de $38 \mathrm{~cm}$ de diâmetro. Foram coletadas dez amostras por ocasião de amostragem, sendo cada uma composta por 50 golpes de rede em movimento de avanço nas diferentes entrelinhas do pomar, excetuando-se as bordas (tamanho amostral por ocasião de coleta $=500$ golpes) .

As amostragens foram mensais durante o período de fevereiro/2001 a janeiro/2002 e realizadas no horário das $10 \mathrm{~h}$ às $14 \mathrm{~h}$. Este horário foi escolhido em razão do acúmulo de orvalho na vegetação durante a noite, que permanece ainda nas primeiras horas da manhã, não permitindo o bom desempenho da rede-de-varredura, que absorve a água e dificulta assim, a retirada dos insetos de seu interior.

A identificação das espécies foi realizada através de chaves dicotômicas (MARUCCI et al., 1999, 2002) e por comparação direta com exemplares do Museu de Ciências e Tecnologia da Pontifícia Universidade Católica do Rio Grande do Sul (MCTP). O material estudado está depositado nas coleções da Faculdade de Agronomia da Universidade Federal do Rio Grande do Sul (FASE), do Museu "Ramiro Gomes da Costa" da Fundação Estadual de Pesquisa Agropecuária do Rio Grande do Sul (FEPAGRO) (MRGC), do Museu de Ciências e Tecnologia da Pontifícia Universidade Católica do Rio Grande do Sul (MCTP) e do Museu de Ciências Naturais da Fundação Zoobotânica do Rio Grande do Sul (MCNZ).

Para a avaliação da confiabilidade da amostragem, foi calculado o Coeficiente de variabilidade da amostra (C.v.a. $)=\left(\mathrm{s}^{\prime} / \mathrm{x}^{\prime}\right)$. 100, onde s' $=$ desvio padrão estimado da amostra (erro padrão) e x' = média estimada das amostras, segundo MüHLEnBERG (1993).

A constância foi medida de duas maneiras: 1) a relação entre o número de amostras nas quais a espécie ocorreu (nA) e o número total de unidades amostrais coletadas $(120) ; 2)$ a relação entre o número de coletas em que a espécie ocorreu (nC) e o número de ocasiões de amostragem (12). A partir dos resultados, foram estabelecidas categorias de constância das espécies, segundo SiLVEIRA Neto (1976), onde: C>50\% - Constante, $50>C>25$ - Acessória e C $<25 \%$ - Acidental.

- A temperatura do ar foi medida em campo, na ocasião das amostragens. Os dados relativos à precipitação pluviométrica foram obtidos junto ao Instituto Nacional de Meteorologia (INMET).

As aplicações de biofertilizante (super-magro) ocorreram nos meses de julho e agosto/2001 e a calda bordalesa foi utilizada em outubro e novembro/2001.

\section{RESULTADOS E DISCUSSÃO}

O tamanho amostral foi testado através do Coeficiente de variabilidade da amostra (C.v.a.) (MüHLENBERG, 1993), para cada ocasião de amostragem, o qual apresentou seu maior valor no mês de abril $(17,6 \%)$. OTT \& CARVALHO (2001) obtiveram a média de $16,8 \%$ para o C.v.a. de cigarrinhas de campos com o mesmo tamanho amostral para 44 coletas. Neste estudo, foi obtida a média de $12,9 \%$ para um total de 12 coletas, demonstrando que o número de unidades amostrais foi satisfatório para a boa caracterização da comunidade.

De um total de 3.333 auquenorrincos (Hemiptera, Auchenorrhyncha) coletados, foram registrados 928 espécimens pertencentes a nove espécies de cicadelíneos reconhecidamente vetores ou considerados potenciais vetores de X. fastidiosa: oito Cicadellini-B. xanthophis, Diedrocephala variegata (Fabricius, 1775), Hortensia similis (Walker, 1851), M. leucomelas, Parathona gratiosa (Blanchard, 1840), Plesiommata corniculata Young, 1977, Sibovia sagata (Signoret, 1854) e Sonesimia grossa (Signoret, 1854), e um Proconiini, Tapajosa rubromarginata (Signoret, 1855).

Dentre as 11 espécies comprovadamente vetoras de $X$. fastidiosa no Estado de São Paulo (FUNDECITRUS, 2004), foram registradas B. xanthophis, M. leucomelas, P. gratiosa, $P$. corniculata e $S$. grossa. As demais espécies de cicadelíneos coletadas neste estudo figuram entre as consideradas potenciais vetoras de X. fastidiosa. Em levantamento com rede-de-varredura na vegetação rasteira de pomares de $C$. sinensis var. Pêra no litoral norte da Bahia, Miranda (2003) registrou apenas $B$. xanthophis e Ferrariana trivittata (Signoret, 1854) entre as cigarrinhas comprovadamente vetoras de $X$. fastidiosa.

Os cicadelíneos mais abundantes foram $H$. similis, $M$. leucomelas e $S$. grossa, que juntas representaram $56,9 \%$ dos indivíduos amostrados (Tab. I). A análise da 
abundância das espécies registradas nas amostras e nas coletas, permitiu sua classificação em categorias de constância. Desta forma, $H$. similis e $M$. leucomelas foram constantes, B. xanthophis, D. variegata, $P$. corniculata, $S$. sagata e $S$. grossa foram acessórias e $P$. gratiosa e $T$. rubromarginata de ocorrência acidental (Tab. I). Se compararmos estes resultados aos obtidos por YАмАмото \& Gravena (2000), constata-se que $H$. similis e $M$. leucomelas foram as únicas espécies constantes nos dois trabalhos. A primeira é considerada uma espécie típica da vegetação herbácea, ocorrendo em menor abundância na planta cítrica (PAIVA et al., 1996). Plesiommata corniculata apresentou ocorrência acessória neste estudo, enquanto em São Paulo mostrou-se constante, provavelmente por naquele estado ser encontrada principalmente nas gramíneas Cynodon dactylon (L.) Pers. (grama-seda) e Brachiaria decumbens Stapf. (capimbraquiária) (MARUCCI et al., 2002), que não são predominantes no pomar de Montenegro. Algumas espécies consideradas acessórias em Montenegro, RS (B. xanthophis e S. grossa), em São Paulo foram de ocorrência acidental, segundo dados de Yамамото \& GRAVENA (2000). Estes autores atribuíram este resultado à amostragem com coletor de sucção motorizado. De acordo com PuRCELl \& ElKingTON (1980), o coletor de sucção não seria o método mais indicado para estabelecer a abundância de cigarrinhas na vegetação herbácea de pomares.

De acordo com a flutuação populacional dos cicadelíneos (Fig. 1), observa-se que a comunidade apresentou maior abundância durante a primavera e o verão, corroborando o período de maior abundância destes insetos registrados em pomares cítricos no Estado de São Paulo (FUNDECITRUS, 2004). Este padrão de abundância ocorre em função do clima quente e seco registrado nestas estações, além de coincidir com a época de brotação das plantas cítricas, fase fenológica preferida como alimento por grande número de espécies de cicadelíneos.

As cigarrinhas foram capturadas em menor número em abril devido às médias de pluviosidade registradas neste mês terem sido superiores em torno de $125 \mathrm{~mm}$ do considerado normal (Mota \& Agendes, 1986). Sabidamente o efeito das mudanças climáticas sobre a dinâmica populacional de insetos, sobretudo a pluviosidade, pode ter efeito negativo nestas populações, em especial sobre os fitófagos (OTTE \& JoERn, 1975; Pollard, 1984; Wallner, 1987). A influência da pluviosidade sobre populações de auquenorrincos foi discutida por OTT \& CARVALHO (2001), que atribuíram o reduzido número de cigarrinhas coletadas com a mesma metodologia no outono em ecossistema de campos nativos ao baixo índice pluviométrico registrado neste período. BOINSKY \& FOWLER (1989) constataram o menor número de "homópteros" capturados com rede-devarredura no final da temporada de chuvas, onde a pluviosidade registrada no período foi superior à média normal do período. A influência da pluviosidade na redução da abundância de auquenorrincos, evidenciada neste trabalho, corrobora as conclusões dos autores supra-citados.

Em dezembro foi registrada a segunda menor densidade de cigarrinhas em decorrência da estrutura da vegetação que apresentou altura em torno de $1,5 \mathrm{~m}$, prejudicando a captura destes insetos pela rede-devarredura, a qual elege as cigarrinhas que habitam as camadas superiores da vegetação. DeLong (1932) em seu trabalho pioneiro sobre o desempenho da rede-de-

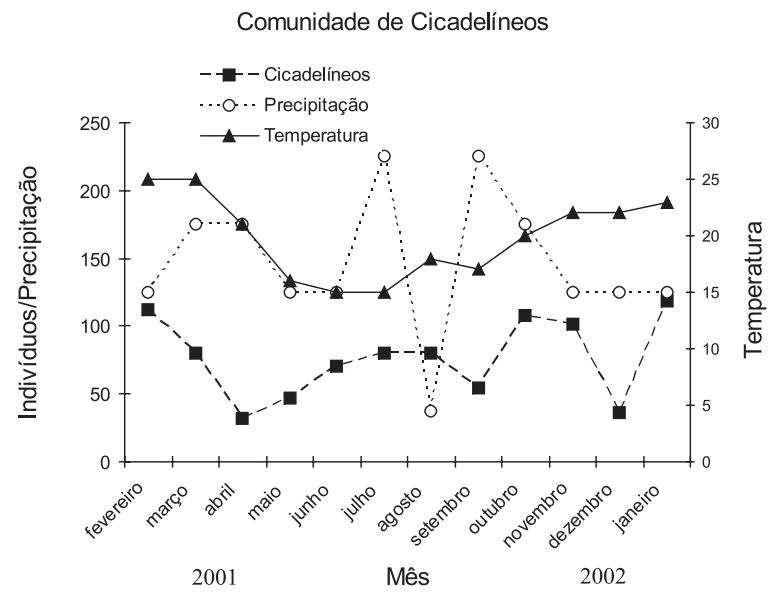

Fig. 1. Flutuação populacional da comunidade de cicadelíneos coletados com rede-de-varredura na vegetação herbácea de pomar de laranja doce no município de Montenegro, RS, no período de fevereiro/2001 a janeiro/2002.

Tabela I. Constância de nove espécies de cicadelíneos coletados com rede-de-varredura na vegetação herbácea de pomar de laranja doce no município de Montenegro, RS, no período de fevereiro/2001 a janeiro/2002 (NA, número de amostras em que a espécie foi registrada; NI, número total de indivíduos coletados da espécie; \% NA = NA/número total de amostras coletadas $\mathrm{x} 100 ; \% \mathrm{NI}=\mathrm{NI} /$ número total de cicadelíneos coletados x 100).

\begin{tabular}{|c|c|c|c|c|c|}
\hline Espécies & NA & $\% \mathrm{NA}$ & Constância & NI & $\% \mathrm{NI}$ \\
\hline Bucephalogonia xanthophis & 45 & 37,50 & Acessória & 84 & 9,05 \\
\hline Diedrocephala variegata & 39 & 32,50 & Acessória & 68 & 7,33 \\
\hline Hortensia similis & 64 & 53,33 & Constante & 227 & 24,46 \\
\hline Macugonalia leucomelas & 66 & 55,00 & Constante & 179 & 19,29 \\
\hline Parathona gratiosa & 5 & 4,17 & Acidental & 8 & 0,86 \\
\hline Plesiommata corniculata & 35 & 29,17 & Acessória & 106 & 11,42 \\
\hline Sibovia sagata & 49 & 40,83 & Acessória & 85 & 9,16 \\
\hline Sonesimia grossa & 56 & 46,67 & Acessória & 122 & 13,15 \\
\hline Tapajosa rubromarginata & 25 & 20,83 & Acidental & 49 & 5,28 \\
\hline Total & 120 & 100 & - & 928 & 100 \\
\hline
\end{tabular}


varredura na amostragem de populações de insetos, já afirmava que a eficiência deste método estava estreitamente relacionado à altura da vegetação. Esta relação foi evidenciada, dentre outros, por NovotNÝ (1992), que estudou a distribuição vertical de cigarrinhas em pradarias, enfatizando que a eficiência da rede-devarredura (número de indivíduos coletados) era intimamente dependente da estrutura da vegetação e de sua altura. Além dos autores citados anteriormente, AndrzejewsKa (1965), Morris (1971, 2000), bem como o presente estudo, registraram menor captura de cigarrinhas em áreas com vegetação alta.

A sazonalidade das espécies de ocorrência constante durante as amostragens (Fig. 2) demonstra que Hortensia similis foi a espécie de maior abundância e constância durante as amostragens, confirmando seu status de típica da vegetação herbácea (PAIVA et al., 1996). De acordo com a longevidade desta espécie em condições de laboratório, 62 a 65 dias (VEGA, 2005), os picos registrados refletem o incremento de novos adultos na população. Os menores registros ocorridos em maio e dezembro podem ser atribuídos, respectivamente, aos efeitos ocasionados pela precipitação pluviométrica atípica registrada em abril, com chuvas em torno de 125 $\mathrm{mm}$ acima do normal para este período, e pela baixa eficiência da rede-de-varredura. Macugonalia leucomelas apresentou distribuição constante ao longo do ano, com maior abundância no verão, estação na qual destacou-se pela presença de 70 indivíduos em janeiro, correspondendo a 39\% do total registrado para esta espécie. Este incremento na população provavelmente tenha ocorrido pela predominância de uma espécie vegetal com altura em torno de $1,5 \mathrm{~m}$ a qual teria atuado como hospedeira preferencial de $M$. leucomelas, podendo suportar maiores densidades destes indivíduos do que a vegetação rasteira, como sugerem os estudos de BROwN et al. (1992) e Waloff \& Solomon (1973).

Em relação à flutuação populacional das espécies consideradas acessórias (Fig. 3), Bucephalogonia xanthophis apresentou maior abundância na primavera

\section{Espécies Constantes}

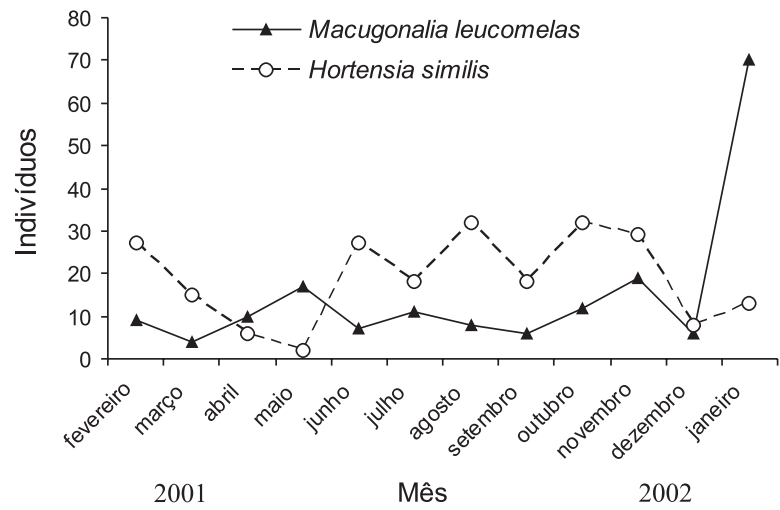

Fig. 2. Flutuação populacional das espécies de cicadelíneos consideradas constantes em coletas com rede-de-varredura na vegetação herbácea de pomar de laranja doce no município de Montenegro, RS, no período de fevereiro/2001 a janeiro/2002. e verão, refletindo o padrão encontrado neste estudo para a comunidade de cicadelíneos e corroborando os estudos da FUNDECITRUS (2004). Diedrocephala variegata, diferentemente das demais espécies, apresentou maior abundância durante o inverno (julho e agosto). Este padrão não foi relacionado a efeitos climáticos ou florísticos e, em razão do desconhecimento da bioecologia desta espécie, não foi possível maior discussão. Em abril, D. variegata não foi registrada em razão da alta precipitação pluviométrica que restringiu a abundância da comunidade de cicadelíneos em geral. Plesiommata corniculata apresentou maior abundância em março e nos três meses seguintes somou apenas 11 espécimes. Em julho e agosto houve um incremento da sua população, a qual voltou a decrescer nos três meses seguintes, não sendo registrado nenhum indivíduo em dezembro de 2001 e janeiro de 2002. A sazonalidade desta espécie também corrobora os efeitos negativos da pluviosidade e a altura da vegetação como fatores determinantes na abundância das cigarrinhas. Sibovia sagata apresentou maior abundância em outubro, acompanhando o padrão de maior abundância de cicadelíneos na primavera e verão. Sonesimia grossa evidenciou maior abundância nos meses de junho e setembro; o seu pico populacional no inverno deveu-se ao excesso de chuvas no outono, que restringiu a abundância das cigarrinhas neste período, fazendo com que a população voltasse a se estabelecer em condições mais favoráveis, em junho, com o registro de temperatura alta para a estação $\left(23,3^{\circ} \mathrm{C}\right)$.

O período de maior abundância dos cicadelíneos no pomar de laranja doce em Montenegro foi na primavera e no verão. Entre as espécies vetoras ou potencialmente vetoras de $X$. fastidiosa, apenas $H$. similis e M. leucomelas apresentaram ocorrência constante durante o período de amostragem.

A comunidade de cigarrinhas no pomar de laranja doce apresentou flutuação sazonal determinada, aparentemente, pelo regime de chuvas, sendo que seu excesso ou escassez traduziram-se em efeitos supressivos

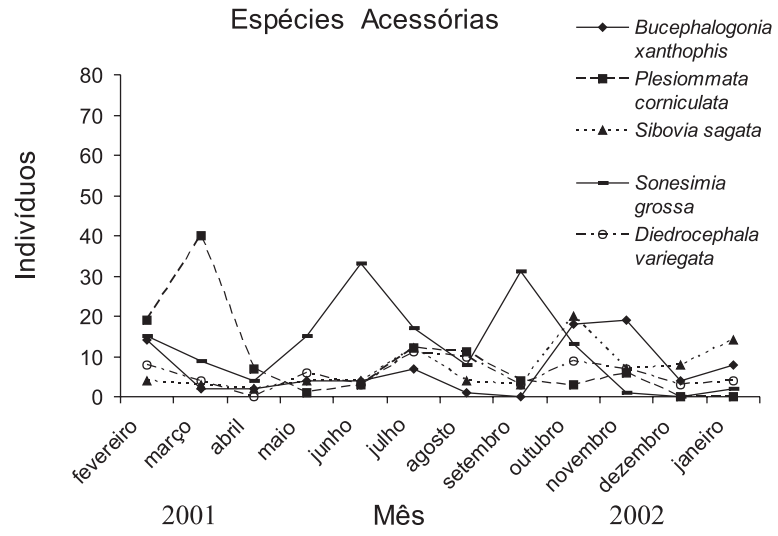

Fig. 3. Flutuação populacional das espécies de cicadelíneos consideradas acessórias em coletas com rede-de-varredura na vegetação espontânea de pomar de laranja doce no município de Montenegro, RS, no período de fevereiro/2001 a janeiro/2002. 
sobre abundância destes insetos. A estrutura da vegetação (altura) também foi um fator relevante na abundância da comunidade, tendo restringido a eficiência da rede-de-varredura em pelo menos uma ocasião de amostragem. Apesar do evidenciado aqui, deve-se salientar que ainda são necessários estudos para que se possa conhecer o real papel destes fatores na organização estrutural destas comunidades. Além disto, outras variáveis como o curto período de tempo de amostragem e a ausência de réplicas requerem prudência na extrapolação do padrão de abundância aqui registrado para outros pomares de laranja doce no Rio Grande do Sul. Desta forma, sugere-se que futuras investigações contemplem estudos que relacionem a influência de fatores abióticos e da estrutura da vegetação sobre comunidades de cicadelíneos em pomares cítricos.

Agradecimentos. Aos acadêmicos de Ciências Biológicas da PUCRS Felipe Rossoni, Otávio Ramos, Isabel Ely, Ricardo Bins Neto e Ana Carolina Anés pelo auxílio na triagem e montagem dos espécimens. À CAPES, FAPERGS e ao PPG Fitotecnia da UFRGS, pelo auxílio financeiro. À Dra. Vera Regina dos Santos Wolff (FEPAGRO), Dr. Andreas Köhler (UNISC) e Dr. Rogério Fernando Pires da Silva (UFRGS) pelas sugestões. Em especial ao Dr. Ricardo Ott (MCN/FZB) e aos dois consultores anônimos pela revisão e críticas ao manuscrito.

\section{REFERÊNCIAS BIBLIOGRÁFICAS}

ANDRZEJEwSKA, L. 1965. Stratification and its dynamics in meadow communities of Auchenorryncha (Homoptera). Ekologia Polska 13:685-715.

Boinsky, N. \& Fowler, N. L. 1989. Seasonal patterns in a tropical lowland forest. Biotropica 21(3):223-233.

Brown, V. K.; Gibson, C. W. D. \& Kathirithamby, J. 1992. Community organization in leafhoppers. Oikos 65:97-106.

DeLong, D. M. 1932. Some problems encountered in the estimation of insect populations by the sweeping method. Annals of the Entomological Society of America 25:13-17.

FUNDECITRUS. 2004. Manual de CVC. Disponível em: <http://www.fundecitrus.com.br>. Acesso em: 11.05.2005.

Huang, G. F. \& Chiaradia, L. A. 1998. Clorose variegada dos citros: caracterização e alternativas no manejo da doença. Agropecuária Catarinense 11(4):52-55.

Marucci, R. C.; Cavichioli, R. R. \& Zucchi, R. 1999. Chave para as espécies de cigarrinhas (Hemiptera:Cicadellidae:Cicadellinae) vetoras da Clorose Variegada dos Citros (CVC). Anais da Sociedade Entomológica do Brasil 28(3):430-446.

2002. Espécies de cigarrinhas (Hemiptera, Cicadellidae, Cicadellinae) em pomares de citros da região de Bebedouro, SP, com descrição de uma espécie nova de Acrogonia Stal. Revista Brasileira de Entomologia 46(2):149-164.

Miranda, M. P. 2003. Levantamento de cigarrinhas (Hemiptera: Cicadellidae) vetoras de Xylella fastidiosa em pomares cítricos do Litoral Norte da Bahia. Dissertação, Escola Superior de Agricultura "Luiz de Queiroz" USP, Piracicaba, nov. 2003. 63p. Disponível em: <http:// www.teses.usp.br/teses/disponiveis/11/11146/tde/08032004- 155606/publico/marcelo.pdf>. Acesso em:10.05.2005.

Morris, M. G. 1971. Differences between the invertebrate faunas of grazed and ungrazed chalk grassland. IV. Abundance and diversity of Homoptera: Auchenorrhyncha. Journal of Applied Ecology 8:37-52.

2000. The effects of structure and its dynamics on the ecology and conservation of arthropods in British grasslands. Biological Conservation 95:129-142.

Mota, F. S. \& Agendes, M. O. O. 1986. Clima e agricultura no Brasil. Porto Alegre, Sagra. 100p.

Mühlenberg, M. 1993. Freilandökologie. Heidelberg, Quelle \& Meyer. 512p.

NovotnÝ, V. 1992. Vertical distribution of leafhoppers (Hemiptera, Auchenorrhyncha) within a meadow community. Acta Entomologica Bohemoslovaca 89:13-20.

Otт, A. P. \& Carvalho, G. S. 2001. Comunidade de cigarrinhas (Hemiptera: Auchenorrhyncha) de uma área de campo do município de Viamão, Rio Grande do Sul, Brasil. Neotropical Entomology 30(2):233-243.

OtTe, D. \& JoERn, A. 1975. Insect territoriality and its evolution: population studies of desert grasshoppers on creosote bushes. Journal of Animal Ecology 44:29-54.

Paiva, P. E. B.; Silva, J. L.; Gravena, S. \& Y амamoto, P. T. 1996. Cigarrinhas de xilema em pomares de laranja do Estado de São Paulo. Laranja 17(1):41-54.

Pollard, E. 1984. Fluctuations in the abundance of butterflies, 1976-82. Ecological Entomology 9:179-188.

Purcell, A. H. \& Elkington, J. S. 1980. A comparison of sampling methods for leafhopper vectors of X-disease in California cherry orchards. Journal of Economic Entomology 73:854-860.

Redak, R. A.; Purcell, A. H.; Lopes, J. R. S.; Blua, M. J.; Mizell III, R. F. \& Andersen, P. C. 2004. The biology of xylem fluidfeeding insect vectors of Xylella fastidiosa and their relation to disease epidemiology. Annual Review of Entomology 49:243-270.

Roberto, S. R. \& Yамамото, P. T. 1998. Flutuação populacional e controle químico de cigarrinhas em citros. Laranja 19(2):269-284.

Rossetti, V.; Gonzalez, M. A. \& Donadio, L. C. 1997. Histórico. In: Donadio, L. C. \& Moreira, C. S. eds. Clorose Variegada dos Citros. Bebedouro, Estação Experimental de Citricultura. p. $1-21$.

Silveira Neto, S. 1976. Manual de Ecologia dos Insetos. São Paulo, Ceres. 419p.

Vega, I. G. 2005. Biología de Hortensia similis Walker (Homoptera: Cicadellidae) bajo condiciones de laboratorio. Disponível em: <http://www.up.ac.pa/facultades/ fcnet/publicados/Res_v3_biologia_3.pdf>. Acesso em:10.05.2005.

WALLNER, W. E. 1987. Factors affecting insect population dynamics: differences between outbreak and non-outbreak species. Annual Review of Entomology 32:317-340.

Waloff, N. \& Solomon, M. G. 1973. Leafhoppers (Auchenorrhyncha: Homoptera) of acidic grassland. Journal of Applied Ecology 10:189-212.

Yамамото, P. T. 1996. Cigarrinhas em citros no Estado de São Paulo. Laranja 17(1):237-239.

Yamamoto, P. T. \& Gravena, S. 2000. Espécies e Abundância de Cigarrinhas e Psilídeos (Homoptera) em Pomares Cítricos. Annais da Sociedade Entomológica do Brasil 29(1):169-176. Yamamoto, P. T.; Roberto, S. R.; Dallapria, J. R. W.; FelipPe, M. R. \& Freitas, E. P. 2002. Espécies e flutuação populacional de cigarrinhas em viveiro de citros no município de Mogi-Guaçu, SP. Revista Brasileira de Fruticultura 24(2):389-394.

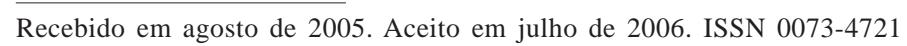

Artigo disponível em: www.scielo.br/isz

Iheringia, Sér. Zool., Porto Alegre, 96(4):425-429, 30 de dezembro de 2006 